\title{
Contraception at extremes of ages, adolescents and special situations
}

\section{Ruchira Nautiyal*, Priyanka Goyal}

\author{
Department of Obstetrics and Gynaecology, Himalayan Institute of Medical Sciences, Dehradun, Uttarakhand, India
}

Received: 15 September 2016

Accepted: 08 October 2016

\author{
*Correspondence: \\ Dr. Ruchira Nautiyal, \\ E-mail: drnauty1@rediffmail.com
}

Copyright: ( ) the author(s), publisher and licensee Medip Academy. This is an open-access article distributed under the terms of the Creative Commons Attribution Non-Commercial License, which permits unrestricted non-commercial use, distribution, and reproduction in any medium, provided the original work is properly cited.

\begin{abstract}
Contraception is designed to prevent pregnancy. Selection of contraceptive depends on many factors including a woman's overall health, age, frequency of sexual activity, number of sexual partners, desire to have children in future, and family history of certain diseases. Hence, planning of pregnancy is of utmost importance to the woman who has in addition a high risk factor. Pregnancy may worsen the preexisting disease or medicines taken for it and vice versa. Therefore, preplanned pregnancy is required with adequate control of high risk factor to ensure optimal pregnancy outcome.
\end{abstract}

Keywords: Contraception, High risk cases, Pregnancy

\section{INTRODUCTION}

Contraception is the prevention of pregnancy. Decision of pregnancy is of utmost importance to the woman who has in addition a high risk factor. That high risk factor could be extremes of ages- adolescents and peri menopausal women; lactating mothers; woman with medical disorders like-diabetes mellitus, hypertension, cardiovascular diseases, liver disorders, HIV and AIDS, epilepsy, migraine, obesity, previous cesarean, gestational trophoblastic disease, breast cancer, endometrial cancer, ovarian cancer and cervical cancer. While prescribing contraception to a high risk women two aspects assume great significance - firstly contraception is given for preplanned pregnancy with optimal control of risk factor to get fruitful results; secondly the alteration in contraceptive efficacy by pathophysiology of disease or by drug.

Medical eligibility criteria (MEC) categories for contraceptive eligibility:

1. A condition for which there is no restriction for the use of the contraceptive method.
2. A condition where the advantages of using the method generally outweigh the theoretical or proven risks.

3. A condition where the theoretical or proven risks usually outweigh the advantages of using the method.

4. A condition which represents an unacceptable health risk if the contraceptive method is used. ${ }^{1}$

\section{Choice of contraception for adolescents}

Adolescence is the period of life between childhood and maturity. WHO has defined adolescents as persons in the 10-19 years age group. Sexual activity and child bearing early in life carry significant risks all over the world. Teenaged mothers face twice the risk of dying from childbirth comparative to woman in $20 \mathrm{~s}$, and their children are more vulnerable to health risks as well. ${ }^{2}$ Counselling regarding HIV, STIs and risks of unwanted pregnancy is of utmost importance as they are especially susceptible to RTIs/STIs. This group should be appropriately advised for contraceptive method and benefits of delayed first intercourse. Dual protection-the use of condoms along with other effective contraceptive 
methods is recommended in adolescent population to prevent them against STI and unwanted pregnancy.

The American College of Obstetricians and Gynaecologists (ACOG) recommend an initial reproductive health visit at age 13 to 15 years to enable provider assess high risk behaviors of the clients and counsel them on risk reduction. ${ }^{3}$ Pelvic examination in adolescents is not required unless complaining of abnormal discharge, pelvic or abdominal pain, or abnormal bleeding. ${ }^{3}$ Privacy and confidentiality should be maintained during counselling and even without the parent or guardian if necessary so that topics like sexual behavior and use of alcohol or drugs can be assessed. ${ }^{4}$ If adolescents are sexually active they should be encouraged to have testing for STIs ${ }^{5}$ and risk associated with both non coital sexual activity and penetrative intercourse. ${ }^{6}$

Long acting reversible contraception (LARC) methodsimplants, intra uterine devices and injectable progestogens are the first line methods for contraception in adolescents. Etonorgestrel implant can be used, side effect is abnormal bleeding. ${ }^{7}$ Levonorgestrel intra uterine system (LNG IUS) with a lower dose of $13.5 \mathrm{mg}$ is marketed for use in nulliparous women and adolescents because of its smaller size and narrower inserter, side effects are oligomenorrhoea and even amenorrhoea. ${ }^{8}$ Copper IUDs can even be resorted to as an emergency contraceptive method within 120 hours of unprotected intercourse. ${ }^{9}$ Depot medroxy progesterone acetate (DMPA) is MEC category $2^{1}$ due to its variable effect of decreasing bone mineral density. Progestin only pills, low dose combined hormonal contraception, contraceptive patch and contraceptive ring can also be used (MEC category $1^{1}$ ) along with barrier methods (dual protection).

\section{Choice of contraception for perimenopausal women}

Implants and intra uterine device (preferably LNG-IUS) are MEC category $1^{1}$, having the additional benefit of managing abnormal uterine bleeding in this age group. If woman is already taking standard dose OCPs then quantity of estrogen to be reduced and quality of progesterone to be improved by giving low dose newer OCPs (loette, femilon). DMPA also MEC category $2^{1}$ due to potential adverse effects on breast, weight and BMD. Low dose OCPOs can be continued even 1 year after menopause for the purpose of hormone replacement therapy (HRT).

\section{Choice of contraception for lactating women}

Less than 6 wks after birth- IUD MEC- $1^{1}$, POC MEC- $3^{1}$, COCs MEC- $4^{1}$.

6 wks -6 months after birth- IUD MEC- $1^{1}$, COCs MEC$3^{1}$, POC MEC- $1^{1}$.

\section{Choice of contraception in women with smoking}

Combined oral contraceptives are MEC category $3^{1}$ for light smokers (fewer than 15 cigarettes per day) and MEC category $4^{1}$ for heavy smokers (15 or more cigarettes per day) as smoking causes vasoconstriction leading to increased cardio vascular risk. All other contraceptives as progesterone only pills, implants, DMPA, intra uterine devices are MEC category $1^{1}$ and can be offered.

\section{Choice of contraceptives in diabetic women}

Contraceptive to be selected for diabetic should not worsen blood sugar control and lipid profile; should not cause or accelerate underlying vasculopathy, nephropathy, neuropathy and retinopathy. IUD is the contraceptive of choice ${ }^{1}$ provided proper asepsis taken while insertion to reduce chance of infection and with optimal control of blood sugar levels.

Low dose OCPs are MEC category 2 for diabetics without vasculopathy and category 4 for diabetics with vasculopathy. ${ }^{1}$ Progesterone only contraceptives are safe in diabetic women MEC category $2 .{ }^{1}$

\section{Choice of contraceptives in hypertensive women}

IUDs are contraceptive of choice. ${ }^{1}$ For malignant hypertension COCs (combined oral contraceptives) are strongly contraindicated (MEC catehory 4$).{ }^{1}$

\section{Choice of contraceptives in cardio vascular diseases}

For clients highly predisposed to cardiovascular risks, thromboembolic disorders, ischaemic heart disease and valvular heart disease IUDs(utering 330 threadless) are MEC category $1^{1}$ and COCs are category 4. ${ }^{1}$ Low dose triphasic pills can be given in mild hypertension without other risks.

For varicose veins both OCPs and IUDs can be safely prescribed (MEC category $1^{1}$ ). Although for superficial thrombophlebitis, OCPs are categorized asMEC $2 .^{1}$

\section{Choice of contraceptives in liver disorders}

IUDs MEC category $1 .{ }^{1}$ COCs MEC category $4 .{ }^{1}$

\section{Choice of contraceptives in HIV and AIDS}

Copper containing IUD MEC category $1^{1}$ in all cases. Women divided into three groups

Woman at risk of HIV-Hormonal contraceptives (HC)MEC 1, LNG IUS-MEC 2. ${ }^{1}$

Woman with HIV-Mild stage-HC-MEC $1^{1}$, LNG IUS MEC- $2^{1}$; severe stage-HC MEC- $1^{1}$, LNGIUS MEC-3. ${ }^{1}$ 
Woman on ART-ON nevirapine/efavirenz/protease inhibitors-DMPA MEC- $1^{1}$, other hormonal contraceptives MEC- $2^{1}$, LNG-IUS -according to staging; if on integrase inhibitors/new art (etavirine) - all methods - MEC1. ${ }^{1}$

\section{Choice of contraceptives in women with epilepsy}

Many anti convulsants are folate antagonists - so pre conceptional folate to be given in pre conceptional period. Phenytoin, carbamazepine, phenobarbitone are potent hepatic enzyme CYT P450 inducers-reducing contraceptive efficacy. COC increases bioavailability of lamotrigine. IUD and DMPA safe MEC-2 ${ }^{1}$.Higher dose COC MEC-3. ${ }^{1}$ Implants and POP should not be used due to reduced efficacy; however DMPA can be used.

\section{Choice of contraceptives in women with migraine}

IUD and barrier contraceptives are safe MEC-1. ${ }^{1}$ COC contraindicated MEC- $4^{1}$ in complex migraine (focal migraine). Low dose COC can be given in simple migraine MEC-2. ${ }^{1}$ Progestogens MEC-2.

\section{Choice of contraceptives in women with obesity}

COC MEC-2 (decreased efficacy). LNG-IUS, copper T MEC- $1 .{ }^{1}$ Weight reduction and life style modifications.

\section{Choice of contraceptives in women with chances of drug interactions}

Anti-tubercular drugs like rifampicin, anti-psychotic drugs, anti-retroviral drugs are enzyme inducers-there IUD is preferred.

\section{Choice of contraceptives in gestational trophoblastic disease}

COC MEC- $1^{1}$, IUD MEC-4. ${ }^{1}$

\section{Choice of contraceptives in women with breast cancer}

Copper IUD-contraceptive choice MEC-1 ${ }^{1}$, COC contraindicated MEC- $4^{1}$, in benign conditions all contraceptives can be used.

\section{Choice of contraceptives in women with endometrial, ovarian and cervical cancer}

OCPs reduce the risk of developing endometrial and ovarian cancer while increases the risk of cervical cancer. Usually treatment of these cancers causes sterility. OCPs, POPs, combined injectables, DMPA, NET-EN and norplant are good choices. Use of IUDs should not be initiated in patient with endometrial or ovarian cancer but may be continued if cancer develops during use. IUD MEC- $4{ }^{1}$ for carcinoma cervix as may cause trauma and subsequent bleeding.

\section{CONCLUSION}

Contraception in high risk women should not be taken lightly and needs special care. IUDs are MEC-41 for puerperal sepsis, PID or STDs current or within 3 months, known pelvic tuberculosis, gestational trophoblastic disease, endometrial, ovarian and cervical cancer. Combined oral or injectable contraceptives are MEC-41 in focal migraine, malignant hypertension, current history of ischemic or complicated valvular heart disease, diabetes with vascular complications or duration $>20$ years, active hepatitis, malignant liver tumours, and major surgery or prolonged immobilization. Progesterone only contraceptives are MEC-41in breast cancer.

\section{Funding: No funding sources \\ Conflict of interest: None declared \\ Ethical approval: Not required}

\section{REFERENCES}

1. WHO. Medical eligibility criteria for contraceptive use Fifth edition 2015. http://apps.who.int/iris/bitstream/10665/172915/1/WHO _RHR_15.07_eng.pdf. Accessed on Sept 15, 2016.

2. Chaudhuri SK. Practice of fertility control. A comprehensive manual. Reed Elsevier India Pvt. Ltd: New Delhi. 2012; 7th ed. pp.285.

3. Committee on Adolescent Health Care. ACOG Committee Opinion no. 598: The initial reproductive health visit. Obstet Gynecol. 2014;123(5):1143-7.

4. Ford C, English A, Sigman G. Confidential healthcare for Adolescents: position paper for the society for adolescent medicine. J Adolesc Health. 2004;35(2):1607.

5. Workowski KA, Bolan GA. Sexually transmitted diseases treatment guidelines, 2015. MMWR Recomm Rep. 2015;64(RR-03):1-137.

6. Committee on adolescent Health Care, Committee on Gynecologic Practice. Committee Opinion NO. 582: addressing health risks of noncoital sexual activity. Obstet Gynecol. 2013;122(6):1378-82.

7. Deokar AM, Jackson W, Omar HA. Menstrual bleeding patterns in adolescents using etonogestrel (ENG) implant. Int J Adolesc Med Health. 2011;23(1):75-7.

8. Wu JP, Pickle S. Extended use of the intrauterine device: a literature review and recommendations for clinical practice. Contraception. 2014;89(6):495-503.

9. Turok DK, Godfrey EM, Wojdyla D. Copper T380 intrauterine device for emergency contraception: highly effective at any time in the menstrual cycle. Hum Reprod. 2013;28(10):2672-6.

Cite this article as: Nautiyal R, Goyal P.

Contraception at extremes of ages, adolescents and special situations. Int J Reprod Contracept Obstet Gynecol 2016;5:3651-3. 